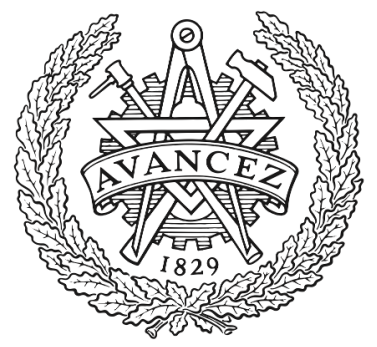

CHALMERS

UNIVERSITY OF TECHNOLOGY

\title{
Online diagnostic ultra-wideband antenna system in high voltage polymeric insulator
}

Downloaded from: https://research.chalmers.se, 2023-04-26 11:37 UTC

Citation for the original published paper (version of record):

Yin, J., Wu, R., Yang, J. et al (2019). Online diagnostic ultra-wideband antenna system in high voltage polymeric insulator. 2019 IEEE International Symposium on Antennas and Propagation and USNC-URSI Radio Science Meeting, APSURSI 2019 - Proceedings: 1725-1726. http://dx.doi.org/10.1109/APUSNCURSINRSM.2019.8888521

N.B. When citing this work, cite the original published paper. 


\section{Online Diagnostic Ultra-Wideband Antenna System in High Voltage Polymeric Insulator}

\author{
Jungang Yin, Runqi Wu \\ College of Electrical and Information Engineering, \\ Hunan University, \\ Changsha, 410082, China \\ yinjungang126@126.com \\ bestldq@foxmail.com
}

\author{
Jian Yang, Xiangdong Xu \\ Department of Electrical Engineering, \\ Chalmers University of Technology, \\ Gothenburg, S412-96, Sweden \\ jian.yang@,chalmers.se \\ xiangdong.xu@chalmers.se
}

\begin{abstract}
A novel ultra-wideband (UWB) antenna system is proposed for fault inspection and online diagnoses on high voltage polymeric insulators. This technique makes use of the multimode transferring theory on dielectric waveguides, where the transmission signal level difference can be employed to detect internal defects in the core rod. An investigation by simulation is performed with regard to the interfacial $\mathrm{mm}$-ordered cracks in transverse and longitudinal orientations, and roughly 30 50 dB and 20 40 dB differences have been observed over the same band 3 10 GHz, respectively.
\end{abstract}

Keywords-online diagnosis; ultra-wideband (UWB) antenna; polymeric insulators; internal defects

\section{INTRODUCTION}

Nowadays, polymeric insulators are widely applied in power systems due to their superior anti-pollution properties, excellent high-dielectric strength, low volume and light weight [1]. Tens of million pieces of polymeric insulators have currently been operating over the world, making up a huge portion of power grid insulators that consist of three categories by materials, i.e., porcelain, glass and polymeric insulators. As time goes by, some internal defects such as interfacial air voids, micro-crack, erosion defect and chemical degradation could gradually appear in polymeric insulators due to various complex circumstances, which will result in severe deterioration on insulation performance [2]-[6]. However, it is of great difficulty to make real time monitoring and diagnoses on the internal defects of polymeric insulators in operation, particularly of outdoor types. An efficient online detection technique is thus called in order to secure reliable operation of polymeric insulators in a smart power grid.

\section{Multimode Transferring THEORY}

A piece of polymeric insulator is generally composed of a polymeric core rod (mainly fiber glass plus epoxy resin), a polymeric (mainly silicon rubber) housing and a number of polymeric (mainly silicon rubber) sheds.

The core rod can be considered as a cylindrical dielectric waveguide. According to the guided wave theory, as illustrated in Fig.1, when a microwave at a proper frequency propagates end-to-end through a normal core rod without any internal defects, the dominant mode $\mathrm{TM}_{01}$ (mode 1) and other higher ordered mode, for example, mode $\mathrm{HE}_{11}$ (mode 2), can be excited within the cylinder, without mutual couplings between the orthogonal modes. However, if there exist some defects inside or on the surface of the core rod, the mutual couplings between the dominate mode and the higher ordered modes will appear with significant values. This is basically the physical principle of the proposed online diagnosis technique.

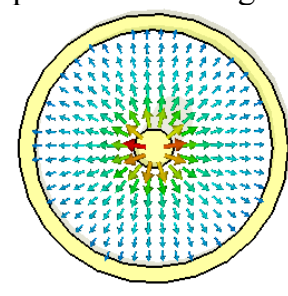

(a)

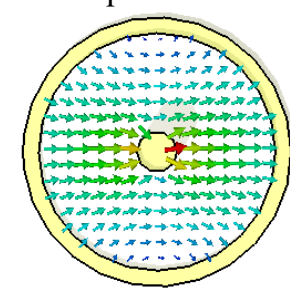

(b)
Fig. 1. Transverse E-field distribution of (a) mode 1 (b) mode 2 in core rod.

\section{SYSTEM DESIGN AND ANALYSES}

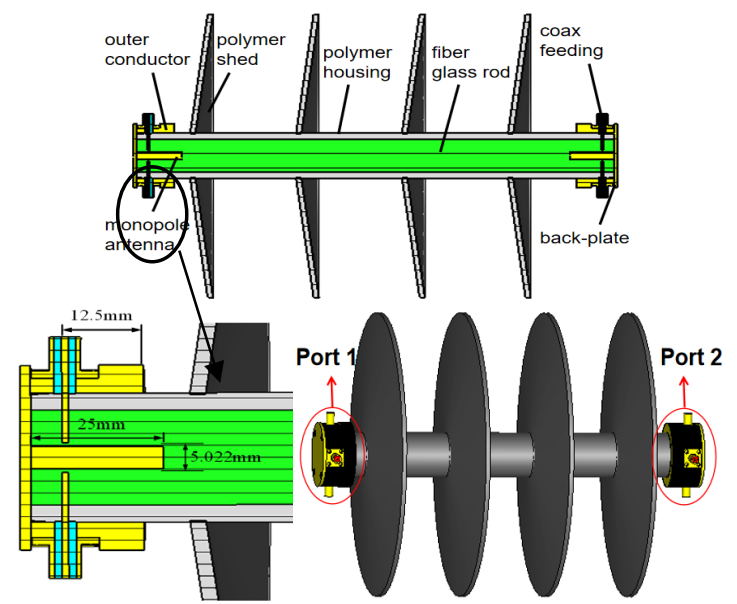

Fig. 2. Geometry and parameters of online diagnostic UWB antenna system.

As shown in Fig. 2, the proposed online diagnostic system mainly consists of two identical antennas that can respectively be integrated in metal (typically aluminum) fittings at the cable end and the ground end, as a transmitter (port 1) and a receiver (port 2), or vice versa. A single antenna is composed of four monopole probes inside the core rod covered by an outer cylindrical conductor that is analogous to a metal cavity with one open aperture. 
Fig. 3 shows how to excite mode 1 and mode 2 microwaves at both ports. Mode 1 can be generated by simultaneously exciting all the four feed probes with equal amplitude and same phase, while mode 2 by exciting two probes for one polarization with equal amplitude but opposite phase

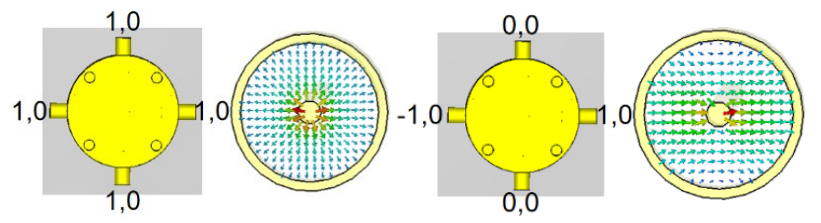

Fig. 3. Port excitations of mode 1 and mode 2 signals within core rod.

\section{SimULATION RESUlTS}

Detection of internal defects in the core rod is investigated by simulation in CST MWS. As depicted in Fig. 4, both transverse (perpendicular to z-axis) and longitudinal (parallel to $z$-axis) conformal cracks on the interface between the core rod and the polymer housing are modelled by air slots, whose volume dimensions are $5.878 \mathrm{~mm}^{*} 1.0 \mathrm{~mm} * 1.0 \mathrm{~mm}$, and $0.5 \mathrm{~mm} * 1.0 \mathrm{~mm} * 10.0 \mathrm{~mm}$, respectively.

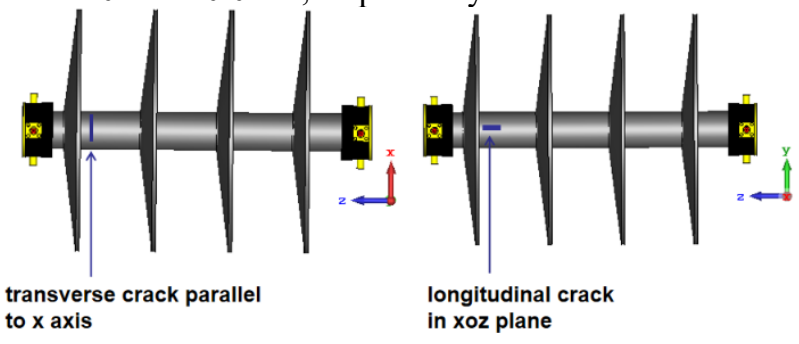

Fig. 4. Transverse and longitudinal cracks on insulator inter-surface.

The simulated transmission S-parameters are exhibited in Fig. 5. As for the transverse cracks, significant difference up to $30 \sim 50 \mathrm{~dB}$ can be observed on the transmission curves $\left(\mathrm{S}_{2(1), 1(2)}\right.$ and $S_{2(2), 1(1)}$ ) between the reference model and the defect model over 3 10 GHz. As for the longitudinal cracks, $20 \sim 40 \mathrm{~dB}$ difference can be observed on the transmission curves between the reference model and the defect model over the same band.
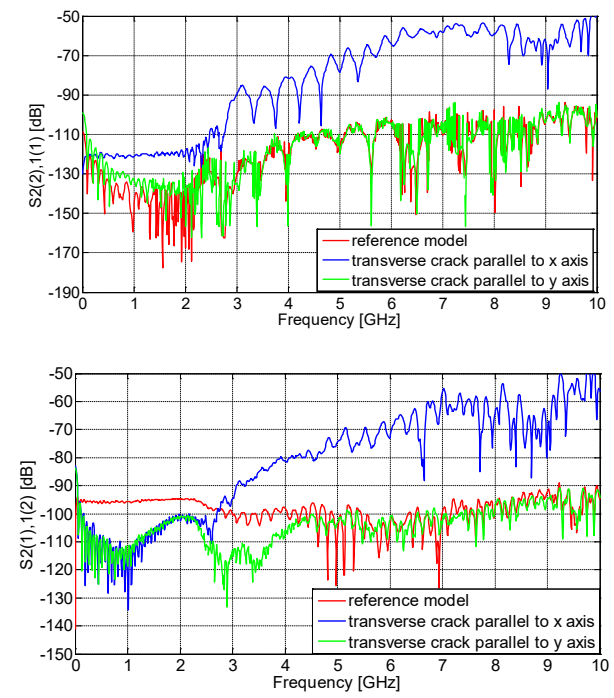
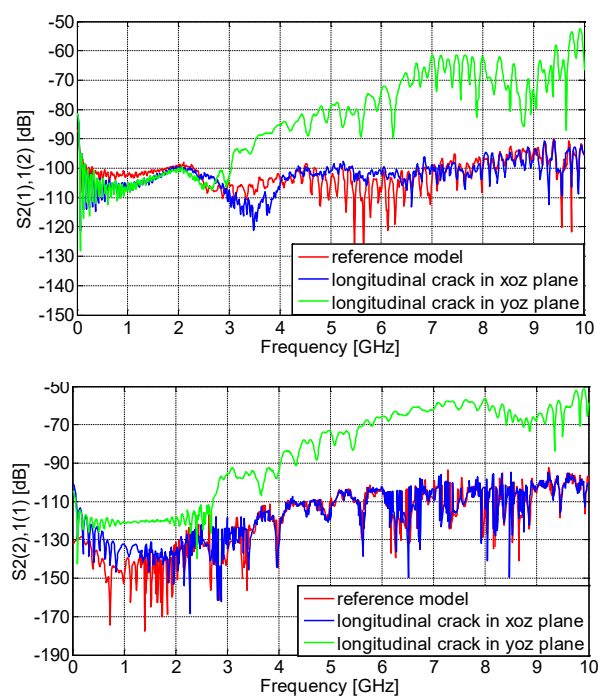

Fig. 5. Simulated S-parameters of reference model and defect model.

\section{CONCLUSION}

A novel online diagnostic UWB antenna system for polymeric insulators is presented in this paper. Detection of interfacial cracks in a dielectric core rod is investigated by simulations. The simulation results show that air cracks in the order of millimeters could successfully be detected by the proposed technique, which may open up a grand new path to fault inspection and online diagnoses on high voltage polymeric outdoor insulators. By the way, an intensive experimental study will be carried out on a manufactured prototype in the near future.

\section{ACKNOWLEDGMENT}

This work was supported in part by the E2 Seed Project of Chalmers University of Technology in Sweden and in part by the Science and Technology Program of Hunan Province in China under grant no. 2018TP2015.

\section{REFERENCES}

[1] C. Yuan, C. Xie, L. Li, F. Zhang and S. M. Gubanski, "Ultrasonic phased array detection of internal defects in composite insulators," in IEEE Transactions on Dielectrics and Electrical Insulation, vol. 23, no. 1, pp. 525-531, February 2016.

[2] B. Lutz, L. Cheng, Z. Guan, L. Wang and F. Zhang, "Analysis of a fractured $500 \mathrm{kV}$ composite insulator - identification of aging mechanisms and their causes," in IEEE Transactions on Dielectrics and Electrical Insulation, vol. 19, no. 5, pp. 1723-1731, October 2012.

[3] M. Kumosa, L. Kumosa and D. Armentrout, "Failure analyses of nonceramic insulators. Part 1: Brittle fracture characteristics," in IEEE Electrical Insulation Magazine, vol. 21, no. 3, pp. 14-27, May-June 2005.

[4] M. Kumosa, L. Kumosa and D. Armentrout, "Failure analyses of nonceramic insulators: part II - the brittle fracture model and failure prevention," in IEEE Electrical Insulation Magazine, vol. 21, no. 4, pp. 28-41, July-Aug. 2005.

[5] J. Andersson, S. M. Gubanski and H. Hillborg, "Properties of interfaces between silicone rubber and epoxy," in IEEE Transactions on Dielectrics and Electrical Insulation, vol. 15, no. 5, pp. 1360-1367, October 2008.

[6] J. Andersson, S. M. Gubanski and H. Hillborg, "Properties of interfaces in silicone rubber," in IEEE Transactions on Dielectrics and Electrical Insulation, vol. 14, no. 1, pp. 137-145, Feb. 2007. 
